\title{
Assessing Robust Stability Properties of Uncertain Genetic Regulatory Networks
}

\author{
Graziano Chesi
}

\begin{abstract}
This paper investigates robust stability properties of genetic regulatory networks (GRNs) affected by uncertainty. Specifically, we consider GRNs with SUM and PROD regulatory functions, where the coefficients are affected polynomially by unknown parameters constrained in a polytope, and where the saturation functions are not exactly known. It is shown that a condition for ensuring that the GRN has a globally asymptotically stable equilibrium point for all admissible uncertainties can be obtained in terms of a convex optimization problem with linear matrix inequalities (LMIs). Moreover, it is shown that a lower bound of the worst-case convergence rate of the trajectories to the equilibrium point over all the admissible uncertainties can be computed by solving a quasi-convex optimization problem with LMIs. The proposed techniques are illustrated by some numerical examples.
\end{abstract}

\section{INTRODUCTION}

Genetic regulatory networks (GRNs) explain the interactions between genes and proteins that are at the basis of the complex mechanisms that regulate biological functions in living organisms. Two basic types of models are used to study GRNs, which are the Boolean model (or discrete model) and the differential equation model (or continuous model), see e.g. [1]-[4] and references therein. In particular, the activity of each gene is expressed in one of two states in Boolean models, while continuous values are used to describe the concentrations of gene products such as mRNAs and proteins in differential equation models.

In GRNs described by differential equation models, the dynamics are expressed through a function of the concentrations of the system, see e.g. [5]-[12] and references therein. This function typically consists of two parts: a linear part which defines the natural decay rate of the considered concentration, and a nonlinear part which describes the influence on the considered concentration by all the other ones. The nonlinear part contains saturation functions, such as the Hill functions, which are combined for example via linear combinations (in this case the GRN is said to have SUM regulatory functions) or products (in this case the GRN is said to have PROD regulatory functions).

GRN models are unavoidably affected by uncertainty. In fact, the coefficients of the model of a GRN cannot be measured exactly due for example to noise. Also, it can be difficult to determine the structure of the saturation functions, which should therefore be considered unknown. This means that the dynamics of the GRN model are affected by uncertain parameters, typically constrained in some bounded

G. Chesi is with the Department of Electrical and Electronic Engineering, University of Hong Kong. Contact information: see http://www.eee.hku.hk/ ${ }^{\circ h e s i ~}$ set of interest. The reader is referred to [13] and references therein for models and techniques typically used to deal with uncertainty in the case of linear systems.

This paper investigates robust stability properties of GRNs with SUM and PROD regulatory functions, where the coefficients are affected polynomially by unknown parameters constrained in a polytope, and where the saturation functions are not exactly known. First, a condition for ensuring that the GRN has a globally asymptotically stable equilibrium point for all the admissible uncertainties is provided in terms of a linear matrix inequality (LMI) feasibility test, which amounts to solving a convex optimization problem. This condition is built by introducing a variable Lyapunov function candidate polynomially parametrized by the uncertainty and by exploiting the square matrix representation (SMR). Second, the problem of estimating the worst-case convergence rate of the trajectories to the equilibrium point over all the admissible uncertainties is considered, which is an important problem in order to establish how fast a GRN can recover from an abnormal state. It is shown that a lower bound of this worstcase convergence rate can be computed by solving a quasiconvex optimization problem. Numerical examples illustrate the use of the proposed techniques. Before proceeding it is worth remarking that this paper extends our previous result in [14] which provides a stability condition for the case of SUM regulatory functions with linear dependence of the coefficients on the uncertain parameters.

The paper is organized as follows. Section II introduces some basic information about GRNs and the problem formulation. Section III derives the proposed results, specifically the condition for robust stability in Section III-A, and the computation of the worst-case convergence rate in Section III-B. Section IV presents some numerical examples that illustrate the use of the proposed techniques. Lastly, Section $\mathrm{V}$ reports some final remarks.

\section{Preliminaries}

\section{A. Uncertain GRNs}

Let us introduce the notation adopted in the paper:

- $\mathbb{R}$ : real number space;

- $\mathbb{R}_{+}$: non-negative real number space;

- 0: null matrix of size specified by the context;

- $I_{n}$ : identity matrix $n \times n$;

- $A^{\prime}$ : transpose of matrix $A$;

- $A>0$ (resp., $A \geq 0$ ): symmetric positive definite (resp., semidefinite) matrix $A$; 
- $\operatorname{diag}(a)$ (resp., $\left.\operatorname{diag}\left(a_{1}, a_{2}, \ldots\right)\right)$ ) diagonal matrix with the entries of vector $a$ (resp., scalars $a_{1}, a_{2}, \ldots$ ) on the diagonal;

- $\operatorname{conv}(a, b, \ldots)$ : convex hull of vectors $a, b, \ldots$;

- $\|a\|$ : Euclidean norm of vector $a$, i.e. $\|a\|=\sqrt{a^{\prime} a}$;

- he $(A): A+A^{\prime}$;

- $A \otimes B$ : Kronecker's product of matrices $A$ and $B$;

- s.t.: subject to.

GRNs described by differential equation models have typically the form

$$
\left\{\begin{aligned}
\dot{m}(t) & =A m(t)+b(p(t)) \\
\dot{p}(t) & =C p(t)+D m(t)
\end{aligned}\right.
$$

where $m=\left(m_{1}, \ldots, m_{n}\right)^{\prime} \in \mathbb{R}_{+}^{n}$ and $p=\left(p_{1}, \ldots, p_{n}\right)^{\prime} \in$ $\mathbb{R}_{+}^{n}$ are vectors containing the mRNA and protein concentrations, $A, C \in \mathbb{R}^{n \times n}$ are negative definite diagonal matrices, $D \in \mathbb{R}^{n \times n}$ is a positive definite diagonal matrix, and $b: \mathbb{R}_{+}^{n} \rightarrow \mathbb{R}_{+}^{n}$ is a nonlinear function.

For instance, in GRNs with SUM regulatory functions the $j$-th entry of $b(p)$ is given by

$$
b_{i}(p)=\sum_{j=1}^{n} \alpha_{i, j} b_{i, j}\left(p_{j}\right)
$$

where the functions $b_{i, j}: \mathbb{R}_{+} \rightarrow \mathbb{R}_{+}$can be expressed as

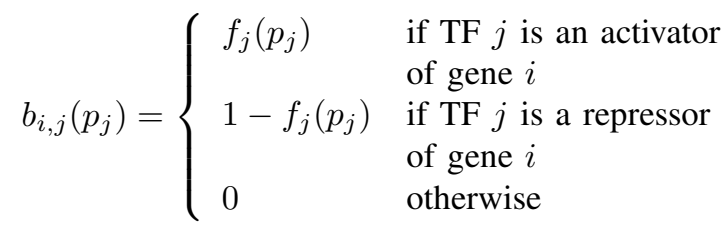

for some saturation functions $f_{j}: \mathbb{R}_{+} \rightarrow \mathbb{R}_{+}$, which typically are chosen of Hill form according to

$$
f_{j}\left(p_{j}\right)=\frac{p_{j}^{h_{j}}}{\beta_{j}^{h_{j}}+p_{j}^{h_{j}}}
$$

for some positive scalars $\beta_{j}$ and $h_{j}$. In GRNs with PROD regulatory functions, $b_{i}(p)$ is a product of functions like $b_{i, j}\left(p_{j}\right)$. In general, hence, $b(p)$ can be expressed as

$$
b(p)=B g(p)+\theta
$$

for some $B \in \mathbb{R}^{n \times l}$ and $\theta \in \mathbb{R}_{+}^{n}$, where the entries of the function $g: \mathbb{R}_{+}^{n} \rightarrow \mathbb{R}_{+}^{l}$ are a subset of the functions $f_{1}\left(p_{1}\right), \ldots, f_{n}\left(p_{n}\right)$ and their products. An example is given by

$$
g(p)=\left(f_{1}\left(p_{1}\right), f_{3}\left(p_{3}\right), f_{1}\left(p_{1}\right) f_{2}\left(p_{2}\right)\right)^{\prime} .
$$

In this paper we consider GRNs as in (1) affected by parametric model uncertainty, in particular

$$
\left\{\begin{aligned}
\dot{m}(t) & =A(u) m(t)+B(u) \phi(p(t))+\theta(u) \\
\dot{p}(t) & =C(u) p(t)+D(u) m(t)
\end{aligned}\right.
$$

where $u \in \mathbb{R}^{q}$ is a time-invariant uncertain vector, and the function $\phi: \mathbb{R}_{+}^{n} \rightarrow \mathbb{R}_{+}^{l}$ account for uncertainty on the function $g(p)$ in (5). We consider that $u$ is constrained in a polytope according to

$$
\begin{aligned}
u & \in \mathcal{U} \\
\mathcal{U} & =\operatorname{conv}\left\{u^{(1)}, \ldots, u^{(r)}\right\}
\end{aligned}
$$

for some $u^{(i)} \in \mathbb{R}^{q}$. The functions of $u$ in (7) are assumed polynomial. Moreover, we consider that $\phi(p)$ is constrained as

$$
\begin{gathered}
\left(\phi_{i}(y+p)-\phi_{i}(p)\right)\left(\sum_{j=1}^{n} \lambda_{i, j} y_{j}-\phi_{i}(y+p)+\phi_{i}(p)\right) \geq 0 \\
\forall p, y \in \mathbb{R}^{n}: p_{i} \geq 0, y_{i} \geq-p_{i} \forall i=1, \ldots, l
\end{gathered}
$$

for some scalars $\lambda_{i, j}$. We denote with $\Phi$ the set of functions $\phi(p)$ satisfying (9), i.e.

$$
\Phi=\{\phi(\cdot): \text { (9) holds }\} .
$$

The problems addressed in this paper are as follows:

1) to establish whether the uncertain GRN (7) has a globally asymptotically stable equilibrium point in $\mathbb{R}_{+}^{n} \times \mathbb{R}_{+}^{n}$ for all $u \in \mathcal{U}$ and for all $\phi(\cdot) \in \Phi$, which is denoted by $\left(m^{*}, p^{*}\right)$;

2) to estimate the worst-case convergence rate of the concentrations, i.e.

$$
\begin{aligned}
\gamma^{*}= & \inf _{u \in \mathcal{U}, \phi(\cdot) \in \Phi} \sup \left\{\gamma \in \mathbb{R}:\|z(t)\|^{2} \leq \xi e^{-\gamma t}\right. \\
& \forall t \geq 0 \text { for some } \xi \in \mathbb{R}\}
\end{aligned}
$$

and

$$
z=\left(\begin{array}{l}
x \\
y
\end{array}\right), \quad x=m-m^{*}, \quad y=p-p^{*} .
$$

Remark 1. Let us observe that the function $\phi(p)$ in (7) can be either known or unknown, and can contain Hill and nonHill saturation functions, provided that condition (9) holds. Also, let us observe that (7) includes GRN models typically adopted in the literature, such as GRNs in SUM form or in PROD form, for instance GRNs in SUM form can be considered by choosing

$$
\phi(p)=\left(f_{1}\left(p_{1}\right), \ldots, f_{n}\left(p_{n}\right)\right)^{\prime} .
$$

\section{B. Square Matrix Representation}

The techniques proposed in this paper exploit forms, i.e. homogeneous polynomials. A form $a(x)$ of degree $d$ in $x \in$ $\mathbb{R}^{n}$ can be written as

$$
a(x)=\sum_{\substack{i_{1}+\ldots i_{n}=d \\ i_{1}, \ldots, i_{n} \geq 0}} a_{i_{1}, \ldots, i_{n}} x_{1}^{i_{1}} \cdots x_{n}^{i_{n}}
$$

for some $a_{i_{1}, \ldots, i_{n}} \in \mathbb{R}$. Forms of even degree can be represented through the square matrix representation (SMR) introduced in [15]. Specifically, let $a(x)$ be a form of degree $2 d$ in $x \in \mathbb{R}^{n}$, and let $b(x) \in \mathbb{R}^{\sigma(n, d)}$ be a vector containing all monomials of degree less than or equal to $d$ in $x$, being $\sigma(n, d)$ given by

$$
\sigma(n, d)=\frac{(n+d-1) !}{(n-1) ! d !} .
$$

Then, $a(x)$ can be written as

$$
a(x)=b(x)^{\prime}(A+L(\alpha)) b(x)
$$


where $A=A^{\prime} \in \mathbb{R}^{\sigma(n, d) \times \sigma(n, d)}$ is such that

$$
a(x)=b(x)^{\prime} A b(x),
$$

$L(\alpha)=L(\alpha)^{\prime}$ is a linear parametrization of

$\mathcal{L}(b(\cdot))=\left\{L=L^{\prime} \in \mathbb{R}^{\sigma(n, d) \times \sigma(n, d)}: \quad b(x)^{\prime} L b(x)=0 \forall x\right\}$

and $\alpha \in \mathbb{R}^{\tau_{1}}$ is a vector of free parameters, being $\tau_{1}$ the dimension of $\mathcal{L}(b(\cdot))$ which is given by

$$
\tau_{1}=\frac{1}{2} \sigma(n, d)(\sigma(n, d)+1)-\sigma(n, 2 d) .
$$

The matrices $A$ and $A+L(\alpha)$ are called SMR matrices of $a(x)$ (also known as Gram matrices). The SMR allows one to establish whether a form $a(x)$ is a sum of squares of polynomials (SOS), i.e.

$$
a(x)=\sum_{i=1}^{k} a_{i}(x)^{2}
$$

for some polynomials $a_{1}(x), \ldots, a_{k}(x)$. Indeed, $a(x)$ is SOS if and only if there exists $\alpha$ satisfying the LMI [15]

$$
A+L(\alpha) \geq 0 \text {. }
$$

Similarly, the SMR can be employed to represent also matrix forms. In particular, a symmetric matrix form $A(x)=$ $A(x)^{\prime} \in \mathbb{R}^{r \times r}$ of degree $2 d$ in $x \in \mathbb{R}^{n}$ can be written as

$$
A(x)=\Delta\left(M_{A}+L(\alpha), b(x), I_{r}\right)
$$

where $\Delta\left(M_{A}+L(\alpha), b(x), I_{r}\right)$ denotes the notation

$$
\begin{aligned}
\Delta\left(M_{A}+L(\alpha), b(x), I_{r}\right)= & \left(b(x) \otimes I_{r}\right)^{\prime}\left(M_{A}+L(\alpha)\right) \\
& \cdot\left(b(x) \otimes I_{r}\right),
\end{aligned}
$$

$M_{A}=M_{A}^{\prime} \in \mathbb{R}^{r \sigma(n, d) \times r \sigma(n, d)}$ is such that

$$
A(x)=\Delta\left(M_{A}, b(x), I_{r}\right),
$$

$L(\alpha)=L(\alpha)^{\prime}$ is a linear parametrization of

$$
\begin{aligned}
\mathcal{L}\left(b(\cdot), I_{r}\right)= & \left\{L=L^{\prime} \in \mathbb{R}^{r \sigma(n, d) \times r \sigma(n, d):}\right. \\
& \left.\Delta\left(L, b(x), I_{r}\right)=0 \forall x\right\}
\end{aligned}
$$

and $\alpha \in \mathbb{R}^{\tau_{2}}$ is a vector of free parameters, being $\tau_{2}$ the dimension of $\mathcal{L}\left(b(\cdot), I_{r}\right)$ which is given by

$$
\tau_{2}=\frac{1}{2} r(\sigma(n, d)(r \sigma(n, d)+1)-(r+1) \sigma(n, 2 d)) .
$$

The SMR allows one to establish whether a symmetric matrix form $A(x)$ is SOS, i.e.

$$
A(x)=\sum_{i=1}^{k} A_{i}(x)^{\prime} A_{i}(x)
$$

for some matrix polynomials $A_{1}(x), \ldots, A_{k}(x)$. Indeed, $A(x)$ is SOS if and only if there exists $\alpha$ satisfying the LMI [16]

$$
M_{A}+L(\alpha) \geq 0 \text {. }
$$

The reader is also referred to [13], [17] for details about SOS polynomials and some of their uses in control systems.

\section{Robustness AnAlysis}

Here we describe the proposed results for robustness analysis. Specifically, we provide a condition for the existence of a globally asymptotically stable equilibrium point for all $u \in \mathcal{U}$ in Section III-A. Then, we address the estimation of the worst-case convergence rate $\gamma^{*}$ in Section III-B.

\section{A. Robust Stability}

Let $d$ be the maximum degree of the matrix polynomials $A(u), \ldots, \theta(u)$, and let us express $A(u)$ as

$$
A(u)=\sum_{i=0}^{d} A^{(i)}(u)
$$

where $A^{(i)}(u)$ is a matrix form of degree $i$ in $u$. Any vector $u \in \mathcal{U}$ can be written as

$$
u=\sum_{i=1}^{r} w_{i} u^{(i)}
$$

for some $w \in \mathcal{W}$ where $\mathcal{W}$ is the simplex:

$$
\mathcal{W}=\left\{w \in \mathbb{R}_{+}^{r}: \sum_{i=1}^{r} w_{i}=1\right\} .
$$

Let us define

$$
\bar{A}(w)=\sum_{i=0}^{d}\left(\sum_{j=1}^{r} w_{j}\right)^{d-i} A^{(i)}\left(\sum_{j=1}^{r} w_{j} u^{(j)}\right) .
$$

We have that $\bar{A}(w)$ is a matrix form of degree $d$ in $w$. Moreover,

$$
\begin{aligned}
& \forall u \in \mathcal{U} \exists w \in \mathcal{W}: A(u)=\bar{A}(w) \\
& \forall w \in \mathcal{W} \exists u \in \mathcal{U}: \quad A(u)=\bar{A}(w) .
\end{aligned}
$$

Similarly, we obtain the matrix forms $\bar{B}(w), \bar{C}(w), \bar{D}(w)$ and $\bar{\theta}(w)$, and hence (7) can be equivalently rewritten as

$$
\left\{\begin{aligned}
\dot{m}(t) & =\bar{A}(w) m(t)+\bar{B}(w) \phi(p(t))+\bar{\theta}(w) \\
\dot{p}(t) & =\bar{C}(w) p(t)+\bar{D}(w) m(t) .
\end{aligned}\right.
$$

Now, let $P(w)=P(w)^{\prime} \in \mathbb{R}^{2 n \times 2 n}$ be a matrix form of degree $\delta$ in $w$, and let us define $Q(w)=Q(w)^{\prime} \in$ $\mathbb{R}^{2 n+l \times 2 n+l}$ as

$$
Q(w)=\text { he }\left(P(w)\left(\begin{array}{ccc}
\bar{A}(w) & 0 & \bar{B}(w) \\
\bar{D}(w) & \bar{C}(w) & 0 \\
0 &
\end{array}\right)\right.
$$

where the " 0 "-blocks are null matrices of suitable size. Also, let $s(w) \in \mathbb{R}^{l}$ be a vector form of degree $\delta+d$ in $w$, and let us define $R(w)=R(w)^{\prime} \in \mathbb{R}^{2 n+l \times 2 n+l}$ as

$$
R(w)=\text { he }\left(\begin{array}{cc}
0 & 0 \\
0 & \Lambda^{\prime} \operatorname{diag}(s(w)) \\
0 & -2 \operatorname{diag}(s(w))
\end{array}\right)
$$

where the "0"-blocks are null matrices of suitable size, and $\Lambda \in \mathbb{R}^{l \times n}$ is the matrix having on its $(i, j)$-th entry the scalar $\lambda_{i, j}$ in condition (9). 
Let $b_{P}(w)$ and $b_{Q}(w)$ be vectors containing all monomials in $w$ of degrees $\delta$ and $\delta+d$, respectively, and let us parametrize $P(w)$ and $s(w)$ as

$$
\begin{aligned}
P(w) & =C_{P}\left(b_{P}(w) \otimes I_{2 n}\right) \\
s(w) & =c_{s}^{\prime} b_{Q}(w)
\end{aligned}
$$

where $C_{P}$ and $c_{s}$ are constants to be determined. Let us introduce the notation

$$
\mathrm{sq}(w)=\left(w_{1}^{2}, \ldots, w_{q}^{2}\right)^{\prime}
$$

and let us express $P(\operatorname{sq}(w), Q(\operatorname{sq}(w))$ and $R(\operatorname{sq}(w))$ as

$$
\begin{aligned}
& P(\operatorname{sq}(w))=\Delta\left(M_{P}, b_{P}(w), I_{2 n}\right) \\
& Q(\operatorname{sq}(w))=\Delta\left(M_{Q}, b_{Q}(w), I_{2 n+l}\right) \\
& R(\operatorname{sq}(w))=\Delta\left(M_{R}, b_{Q}(w), I_{2 n+l}\right)
\end{aligned}
$$

for some matrices $M_{P}=M_{P}^{\prime}, M_{Q}=M_{Q}^{\prime}$ and $M_{R}=M_{R}^{\prime}$. We have the following result.

Theorem 1: The uncertain GRN (7) has a globally asymptotically stable equilibrium point in $\mathbb{R}_{+}^{n} \times \mathbb{R}_{+}^{n}$ for all $u \in \mathcal{U}$ and for all $\phi(\cdot) \in \Phi$ if there exist $C_{P}, c_{s}, \alpha$ and $\beta$ satisfying the LMIs

$$
\left\{\begin{array}{l}
0<M_{P}+L_{P}(\alpha) \\
0>M_{Q}+M_{R}+L_{Q}(\beta)
\end{array}\right.
$$

where $L_{P}(\alpha)$ and $L_{Q}(\beta)$ are linear parametrizations of $\mathcal{L}\left(b_{P}, I_{2 n}\right)$ and $\mathcal{L}\left(b_{Q}, I_{2 n+l}\right)$.

Proof. Let us pre- and post- multiply the first LMI in (40) by $\left(b_{P}(w) \otimes I_{2 n}\right)^{\prime}$ and $b_{P}(w) \otimes I_{2 n}$, respectively. We obtain:

$$
\begin{aligned}
0 & <\left(b_{P}(w) \otimes I_{2 n}\right)^{\prime}\left(M_{P}+L_{P}(\alpha)\right)\left(b_{P}(w) \otimes I_{2 n}\right) \\
& =\Delta\left(M_{P}, b_{P}(w), I_{2 n}\right) \\
& =P(\operatorname{sq}(w))
\end{aligned}
$$

i.e. $P(\operatorname{sq}(w))>0$ for all $w \neq 0$. From Theorem 1.17 in [13] this holds if and only if

$$
P(w)>0 \quad \forall w \in \mathcal{W} .
$$

Let us pre- and post- multiply the second LMI in (40) by $\left(b_{Q}(w) \otimes I_{2 n+l}\right)^{\prime}$ and $b_{Q}(w) \otimes I_{2 n+l}$, respectively. We analogously obtain that

$$
Q(w)+R(w)<0 \quad \forall w \in \mathcal{W} .
$$

Let us define $x, y$ and $z$ as in (12) where $\left(m^{*}, p^{*}\right)$ is an unknown equilibrium point of (34) in $\mathbb{R}_{+}^{n} \times \mathbb{R}_{+}^{n}$. Let us also define the function

$$
v(z, w)=z^{\prime} P(w) z .
$$

One has that the time derivative of $v(z, w)$ along the trajectories of (34) is given by

$$
\dot{v}(\zeta, w)=\zeta^{\prime} Q(w) \zeta
$$

where

$$
\begin{aligned}
\zeta & =\left(z^{\prime}, z_{\phi}^{\prime}\right)^{\prime} \\
z_{\phi} & =\phi\left(y+p^{*}\right)-\phi\left(p^{*}\right)
\end{aligned}
$$

Let us observe that the condition $Q(w)+R(w)<0$ for all $w \in \mathcal{W}$ implies that the entries of $s(w)$ are positive for all $w \in \mathcal{W}$. This fact and the definition of $z_{\phi}$ imply that

$$
\zeta^{\prime} R(w) \zeta \geq 0 \quad \forall w \in \mathcal{W} \forall \phi(\cdot) \in \Phi .
$$

Consequently, one has that

$$
0>\zeta^{\prime}(Q(w)+R(w)) \zeta \geq \zeta^{\prime} Q \zeta
$$

hence implying that $v(x, y, w)>0$ and $\dot{v}(\zeta, w)<0$ for all $(x, y) \neq 0$ for all $w \in \mathcal{W}$ and for all $\phi(\cdot) \in \Phi$. Therefore, $\left(m^{*}, p^{*}\right)$ is a globally asymptotically stable equilibrium point in $\mathbb{R}_{+}^{n} \times \mathbb{R}_{+}^{n}$.

Theorem 1 provides a condition for establishing the existence of a globally asymptotically stable equilibrium point in the GRN (7) for all admissible uncertainties. This condition consists of an LMI feasibility test, which amounts to solving a convex optimization problem, see e.g. [18]. Also, this condition depends on the chosen integer $\delta$. It can be shown that the conservatism of the condition does not increase as $\delta$ increases, moreover there can be cases where the condition is satisfied for $\delta+1$ but it is not for $\delta$. On the other hand, the computational burden increases as $\delta$ increases, and hence a trade-off is necessary when choosing $\delta$.

One way to select $\delta$ consists of starting with the smallest allowed value, i.e. $\delta=0$, and then increasing $\delta$ till either the condition of Theorem 1 is satisfied or the computational burden becomes too large: this is motivated by the fact that the computational burden for $\delta+1$ is always much larger than that for $\delta$. Another way consists of choosing the largest $\delta$ for which the maximum number of scalar variables in the LMI feasibility test (40) (and, hence, the computational burden) is smaller than a specified limit: this allows one obtain the least conservative condition for a selected price without trying different values of $\delta$.

From the biological viewpoint the condition of Theorem 1 allows one to ensure that the concentrations of mRNA and protein converge to some constant values for all admissible uncertainties. This also means that, whenever the condition holds, the GRN has no multiple equilibria and no limit cycles, which may represent an undesired condition for the organism under study such as a disease. See e.g. [19] regarding undesired multiple equilibria.

\section{B. Worst-Case Convergence Rate}

Here we consider the problem of estimating the worstcase convergence rate $\gamma^{*}$ defined in (11). It is known that the convergence rate of the trajectories can be estimated by means of Lyapunov functions, in particular by imposing that the time derivative of the Lyapunov function is smaller than the Lyapunov function scaled by the convergence rate. In our case this translates into the condition

$$
\dot{v}(\zeta, w) \leq-\gamma v(z, w) \quad \forall x, y \forall w \in \mathcal{W} \forall \phi(\cdot) \in \Phi
$$

where $v(z, w)$ and $\dot{v}(\zeta, w)$ are defined as in (44)-(45), and $\gamma$ is the guaranteed convergence rate. In fact, (49) ensures that

$$
v(z(t), w) \leq v(z(0), w) e^{-\gamma t} \quad \forall z(0) \forall t \geq 0 .
$$


However, a difficulty arises: in fact, the condition (49) cannot be expressed via LMIs since both $\gamma$ and $v(z, w)$ are variables. This means that estimating the worst-case convergence rate $\gamma^{*}$ would require the use of bilinear matrix inequalities (BMIs), which unfortunately lead to nonconvex optimizations.

In order to cope with this problem, we proceed as follows. Let us define $S(w)=S(w)^{\prime} \in \mathbb{R}^{2 n+l \times 2 n+l}$ as

$$
S(w)=\left(\begin{array}{cc}
\bar{\tau}(w) P(w) & 0 \\
0 & 0
\end{array}\right)
$$

where the "0"-blocks are null matrices of suitable size, and let us express $S(\mathrm{sq}(w))$ as

$$
S(\operatorname{sq}(w))=\Delta\left(M_{S}, b_{Q}(w), I_{2 n+l}\right)
$$

where $M_{S}=M_{S}^{\prime}$ is a matrix built with respect to the vector $b_{Q}(w)$ used in (39). The following result holds.

Theorem 2: Let us define the quantity

$$
\hat{\gamma}_{\delta}=\frac{1}{\hat{\mu}}
$$

where

$$
\begin{gathered}
\hat{\mu}=\inf _{C_{P}, c_{s}^{(i)}, \alpha, \beta^{(i)}, \mu} \mu \\
\text { s.t. }\left\{\begin{aligned}
0< & M_{P}+L_{P}(\alpha) \\
0< & \mu\left(M_{Q}+M_{R}^{(1)}+L_{Q}\left(\beta^{(1)}\right)\right)+M_{S} \\
& +M_{R}^{(2)}+L_{Q}\left(\beta^{(2)}\right) \\
0< & M_{Q}+M_{R}^{(1)}+L_{Q}\left(\beta^{(1)}\right) \\
0< & \mu
\end{aligned}\right.
\end{gathered}
$$

where $L_{P}(\alpha)$ and $L_{Q}(\cdot)$ are linear parametrizations of $\mathcal{L}\left(b_{P}, I_{2 n}\right)$ and $\mathcal{L}\left(b_{Q}, I_{2 n+l}\right)$, and $M_{R}^{(i)}$ is built as $M_{R}$ by replacing $c_{s}$ with $c_{s}^{(i)}$. Then,

$$
\hat{\gamma}_{\delta} \leq \gamma^{*} .
$$

Proof. Let us suppose that the inequalities in (54) hold. From the first inequality, by proceeding as in the proof of Theorem 1 , one obtains that $P(w)>0$ for all $w \in \mathcal{W}$. Let us preand post- multiply the second inequality in $(40)$ by $\left(b_{Q}(w) \otimes\right.$ $\left.I_{2 n+l}\right)^{\prime}$ and $b_{Q}(w) \otimes I_{2 n+l}$, respectively. We obtain that

$$
\begin{aligned}
0 & \Delta\left(\mu\left(M_{Q}+M_{R}^{(1)}+L_{Q}\left(\beta^{(1)}\right)\right)+M_{S}+M_{R}^{(2)}\right. \\
& \left.+L_{Q}\left(\beta^{(2)}\right), b_{Q}(w), I_{2 n+l}\right) \\
= & \mu\left(Q(\operatorname{sq}(w))+R^{(1)}(\operatorname{sq}(w))\right)+P(\operatorname{sq}(w)) \\
& +R^{(2)}(\operatorname{sq}(w))
\end{aligned}
$$

for all $w \neq 0$, and hence that

$$
Q(w)+R^{(1)}(w)+\frac{1}{\mu}\left(\bar{\tau}(w) P(w)+R^{(2)}(w)\right)<0
$$

for all $w \in \mathcal{W}$. Let us observe that

$$
\zeta^{\prime} R^{(i)} \zeta \geq 0 \quad \forall w \in \mathcal{W} \forall i=1,2 \forall \phi(\cdot) \in \Phi,
$$

and hence

$$
\dot{v}(\zeta, w)+\frac{1}{\mu} v(z, w)<0 \quad \forall w \in \mathcal{W} \forall \phi(\cdot) \in \Phi .
$$

This implies that $\mu^{-1}<\gamma^{*}$, and therefore (55) holds.

Theorem 2 provides a lower bound of the worst-case convergence rate of the concentrations $\gamma^{*}$ in (11). This lower bound is obtained by solving (54), which is a GEVP and belongs to the class of quasi-convex optimizations, see e.g. [18]. It is worth observing that the fourth inequality in (54), which ensures that the obtained lower bound is positive, is not conservative since $\gamma^{*}$ is clearly positive if the GRN (7) is globally asymptotically stable. Moreover, the third inequality in (54) guarantees that the optimization problem is a GEVP, and the variable $\beta^{(1)}$ has the role of exploiting additional degrees of freedom through the matrix $L_{Q}\left(\beta^{(1)}\right)$ in this inequality.

The lower bound provided by Theorem 2 is a function of the chosen $\delta$. It can be shown that the conservatism of this lower bound does not increase as $\delta$ increases, i.e.

$$
\hat{\gamma}_{\delta+1} \geq \hat{\gamma}_{\delta}
$$

The biological implication of Theorem 2 is to provide a guaranteed minimum speed of the convergence of the concentrations of mRNA and protein to their equilibrium values. This allows one to establish how fast the GRN can recover for all admissible uncertainties from an abnormal state.

\section{EXAMPLES}

This section illustrates the use of the proposed techniques with some numerical examples. All the computations are done in Matlab with the toolbox SeDuMi.

\section{A. Example 1}

Let us consider the uncertain GRN with SUM and PROD regulatory functions given by

$$
\left\{\begin{array}{l}
\dot{m}_{1}=-4 m_{1}+1-f_{3}\left(p_{3}\right) \\
\dot{m}_{2}=-2 m_{2}+(1+u)\left(1-f_{1}\left(p_{1}\right)\right) \\
\dot{m}_{3}=-m_{3}+(3-2 u)\left(1-f_{1}\left(p_{1}\right) f_{2}\left(p_{2}\right)\right) \\
\dot{p}_{1}=-p_{1}+m_{1} \\
\dot{p}_{2}=-p_{2}+2 m_{2} \\
\dot{p}_{3}=-p_{3}+3 m_{3}
\end{array}\right.
$$

where the functions $f_{i}\left(p_{i}\right)$ are the Hill functions in (4) with $\beta_{i}=1$ and $h_{i}=2$, and $u$ is an uncertain parameter satisfying

$$
u \in[0,1] .
$$

The problem is to establish whether this GRN has a globally asymptotically stable equilibrium point for all $u \in \mathcal{U}$.

Let us define $w=(u, 1-u)^{\prime}$. This system can be rewritten as in (34) with

$$
\begin{aligned}
\bar{A}(w) & =\left(w_{1}+w_{2}\right) \operatorname{diag}(-4,-2,-1) \\
\bar{B}(w) & =\operatorname{diag}\left(-w_{1}-w_{2},-2 w_{1}-w_{2},-w_{1}-3 w_{2}\right) \\
\bar{C}(w) & =\left(w_{1}+w_{2}\right) \operatorname{diag}(-1,-1,-1) \\
\bar{D}(w) & =\left(w_{1}+w_{2}\right) \operatorname{diag}(1,2,3) \\
\bar{\theta}(w) & =\left(w_{1}+w_{2}, 2 w_{1}+w_{2}, w_{1}+3 w_{2}\right)^{\prime} \\
\phi(p) & =\left(f_{3}\left(p_{3}\right), f_{1}\left(p_{1}\right), f_{1}\left(p_{1}\right) f_{2}\left(p_{2}\right)\right)^{\prime} .
\end{aligned}
$$


The conditions in (9) are simply satisfied by selecting the entries $\lambda_{i, j}$ of $\Lambda$ according to

$$
\Lambda=\left(\begin{array}{ccc}
0 & 0 & 0.650 \\
0.650 & 0 & 0 \\
0.650 & 0.650 & 0
\end{array}\right)
$$

We use Theorem 1 with the simple choice $\delta=0$, and we find that the system has a globally asymptotically stable equilibrium point for all the admissible uncertainties. The LMI feasibility test (40) has 63 scalar variables in this case.

\section{B. Example 2}

Here we consider the repressilator investigated in Escherichia coli [20]:

$$
\left\{\begin{array}{l}
\dot{m}_{i}=-a_{i}^{r e p} m_{i}+b_{i}^{r e p}\left(1-f_{j}\left(p_{j}\right)\right) \\
\dot{p}_{i}=-c_{i}^{r e p}\left(p_{i}-m_{i}\right) \\
i=\text { lacl,tet }, \text { cl } \\
j=\text { cl, lacl, tet } R
\end{array}\right.
$$

where the functions $f_{j}\left(p_{j}\right)$ are the Hill functions in (4) with $\beta_{i}=1$ and $h_{i}=2$, and $a_{i}^{r e p}, b_{i}^{r e p}, c_{i}^{r e p}$ are positive coefficients. Let us consider the following numerical case where some of the coefficients of the repressilator are uncertain:

$$
\begin{array}{ccl}
a_{1}^{r e p}=2, & a_{2}^{r e p}=3, & a_{3}^{r e p}=1 \\
b_{1}^{r e p}=4-3 u, & b_{2}^{r e p}=1, & b_{3}^{r e p}=1+3 u \\
c_{1}^{r e p}=1, & c_{2}^{r e p}=1, & \quad c_{3}^{r e p}=1 \\
& u \in[0,1] .
\end{array}
$$

The problem is to estimate the worst-case convergence rate of the concentrations, i.e. $\gamma^{*}$ in (11).

Let us define $w=(u, 1-u)^{\prime}$. This system can be rewritten as in (34) with

$$
\begin{aligned}
\bar{A}(w) & =\left(w_{1}+w_{2}\right) \operatorname{diag}(-2,-3,-1) \\
\bar{B}(w) & =\operatorname{diag}\left(-w_{1}-4 w_{2},-w_{1}-w_{2},-4 w_{1}-w_{2}\right) \\
\bar{C}(w) & =\left(w_{1}+w_{2}\right) \operatorname{diag}(-1,-1,-1) \\
\bar{D}(w) & =\left(w_{1}+w_{2}\right) \operatorname{diag}(1,1,1) \\
\bar{\theta}(w) & =\left(w_{1}+4 w_{2}, w_{1}+w_{2}, 4 w_{1}+w_{2}\right)^{\prime} \\
\phi(p) & =\left(f_{3}\left(p_{3}\right), f_{1}\left(p_{1}\right), f_{2}\left(p_{2}\right)\right)^{\prime} .
\end{aligned}
$$

The conditions in (9) are simply satisfied by selecting the entries $\lambda_{i, j}$ of $\Lambda$ according to

$$
\Lambda=\left(\begin{array}{ccc}
0 & 0 & 0.650 \\
0.650 & 0 & 0 \\
0 & 0.650 & 0
\end{array}\right)
$$

Let us use Theorem 2. By using $\delta=0$ and $\delta=1$ we find the lower bounds $\hat{\gamma}_{0}=0.114$ and $\hat{\gamma}_{1}=0.409$. In particular, the number of scalar variables in the GEVP (54) is 106 for $\delta=0$ and 382 for $\delta=1$.

\section{CONCLUSION}

This paper has investigated robust stability properties of GRNs with SUM and PROD regulatory functions, where the coefficients are affected polynomially by unknown parameters constrained in a polytope, and where the saturation functions are not exactly known. Specifically, a condition for ensuring the existence of a globally asymptotically stable equilibrium point for all admissible uncertainties has been provided in terms of an LMI feasibility test. Moreover, a lower bound of the worst-case convergence rate of the trajectories to the equilibrium point over all the admissible uncertainties has been derived through the solution of a quasi-convex optimization problem with LMIs.

\section{ACKNOWLEDGEMENT}

The author would like to thank the Associate Editor and Reviewers for their comments. This work is supported in part by the Research Grants Council of Hong Kong (Grant HKU711208E).

\section{REFERENCES}

[1] T. Akutsu, S. Miyano, and S. Kuhara. Inferring qualitative relations in genetic networks and metabolic pathways. Bioinformatics, 16:727$734,2000$.

[2] H. Toh and K. Horimoto. System for automatically inferring a genetic network from expression profiles. Journal of Biological Physics, 28:449-464, 2002.

[3] Y. Wang, T. Joshi, X.-S. Zhang, D. Xu, and L. Chen. Inferring gene regulatory networks from multiple microarray datasets. Bioinformatics, 22(19):2413-2420, 2006.

[4] C. Li, L. Chen, and K. Aihara. A systems biology perspective on signal processing in genetic network motifs. IEEE Signal Processing Magazine, 221(3):136-142, 2007.

[5] L. Chen and K. Aihara. Stability of genetic regulatory networks with time delay. IEEE Trans. on Circuits and Systems I, 49(5):602-608, 2002.

[6] H. Toh and K. Horimoto. Inference of a genetic network by a combined approach of cluster analysis and graphical gaussian modeling. Bioinformatics, 18:287-297, 2002.

[7] T. Akutsu, S. Kuhara, O. Maruyama, and S. Miyano. Identification of genetic networks by strategic gene disruptions and gene overexpressions under a boolean model. Theoretical Computer Science, 298:235251, 2003.

[8] L. Chen, R. Wang, T. Kobayashi, and K. Aihara. Dynamics of gene regulatory networks with cell division cycle. Physical Review E, 70:011909, 2004

[9] C. Li, L. Chen, and K. Aihara. Stability of genetic networks with sum regulatory logic: Lure system and LMI approach. IEEE Trans. on Circuits and Systems I, 53(11):2451-2458, 2006.

[10] Z. Wang, J. Lam, G. Wei, K. Fraser, and X. Liu. Filtering for nonlinear genetic regulatory networks with stochastic disturbances. IEEE Trans. on Automatic Control, 53(10):2448-2457, 2008.

[11] G. Chesi. Computing equilibrium points of genetic regulatory networks. Trans. on Computational Systems Biology XI, LNBI 5750, pages 268-282, 2009.

[12] W. Yu, J. Lu, G. Chen, Z. Duan, and Q. Zhou. Estimating uncertain delayed genetic regulatory networks: an adaptive filtering approach. IEEE Trans. on Automatic Control, 54(4):892-897, 2009.

[13] G. Chesi, A. Garulli, A. Tesi, and A. Vicino. Homogeneous Polynomial Forms for Robustness Analysis of Uncertain Systems. Springer, 2009.

[14] G. Chesi and Y. S. Hung. Stability analysis of uncertain genetic SUM regulatory networks. Automatica, 44(9):2298-2305, 2008.

[15] G. Chesi, A. Tesi, A. Vicino, and R. Genesio. On convexification of some minimum distance problems. In European Control Conf., Karlsruhe, Germany, 1999.

[16] G. Chesi, A. Garulli, A. Tesi, and A. Vicino. Robust stability for polytopic systems via polynomially parameter-dependent Lyapunov functions. In IEEE Conf. on Decision and Control, pages 4670-4675, Maui, Hawaii, 2003.

[17] G. Chesi. LMI techniques for optimization over polynomials in control: a survey. IEEE Trans. on Automatic Control, to appear, 2010.

[18] S. Boyd, L. El Ghaoui, E. Feron, and V. Balakrishnan. Linear Matrix Inequalities in System and Control Theory. SIAM, 1994.

[19] H. Lei, X. Wang, R. Wang, X. Zhu, L. Chen, and J. Zhang. Analysis on steady states of photosynthetic carbon metabolic system. In IEEE Conf. on Decision and Control, pages 5643-5648, Shanghai, China, 2009.

[20] M. B. Elowitz and S. Leibler. A synthetic oscillatory network of transcriptional regulators. Nature, 403:335-338, 2000. 